\title{
The fitter the better? Cardiopulmonary Exercise Testing can predict pulmonary exacerbations in Cystic Fibrosis
}

\author{
Asterios Kampouras ${ }^{1}$, Elpis Hatziagorou ${ }^{2}$, Thomas Kalantzis ${ }^{3}$, Vasiliki Avramidou ${ }^{2}$, \\ Kalliopi Kontouli ${ }^{2}$, Fotis Kirvasilis ${ }^{2}$, and John Tsanakas ${ }^{2}$ \\ ${ }^{1}$ Aristotle University of Thessaloniki Faculty of Health Sciences \\ ${ }^{2}$ Aristotle University of Thessaloniki School of Health Sciences \\ ${ }^{3}$ University of Macedonia School of Information Sciences
}

October 20, 2020

\begin{abstract}
Background: The role of Cardiopulmonary Exercise Testing (CPET) in the assessment of prognosis in CF is crucial. However, as the overall survival of the disease becomes better, the need for examinations that can predict pulmonary exacerbations (PEx) and subsequent deterioration becomes evident. Aim: To evaluate whether CPET derived parameters can be used as prognostic indexes for pulmonary exacerbations in patients with CF. Methods: Data from a 10-year follow up with CPET and spirometry of CF patients were used to perform our analyses. Pulmonary exacerbations were recorded. We used a survival analysis through Cox Regression to assess the prognostic role of CPET parameters for PeX. CPET parameters and other variables such as sputum culture, age, and spirometry measurements were tested via multivariate cox models. Results: During a 10-year period (2009-2019) 78 CF patients underwent CPET. Cox regression analysis revealed that VO2peak\% predicted (Hazard ratio (HR), $0.988(0.975,1.000) \mathrm{p}=0.042)$, PetCO2 ( End-tidal CO2 at peak exercise) (HR $0.948(0.913,0.984) \mathrm{p}=0.005), \mathrm{VE} / \mathrm{VO} 2$, (respiratory equivalent for oxygen at peak exercise) (HR $1.032(1.003,1.062) \mathrm{p}=0.033$ were significant predictors of pulmonary exacerbations in the short term after the CPET. Additionally, patients with VO2peak\% predicted $<60 \%$ had 4,5 times higher relative risk of having a PEx than those with higher exercise capacity. Conclusions: CPET can provide valuable information regarding upcoming pulmonary exacerbation in CF. Patients with VO2peak $<60 \%$ are at great risk of subsequent deterioration. Regular follow up of CF patients with Exercise Testing can highlight their clinical image and direct therapeutic interventions.
\end{abstract}

\section{Hosted file}

main doc CPET.pdf available at https://authorea.com/users/368753/articles/487796-the-fitterthe-better-cardiopulmonary-exercise-testing-can-predict-pulmonary-exacerbations-incystic-fibrosis 


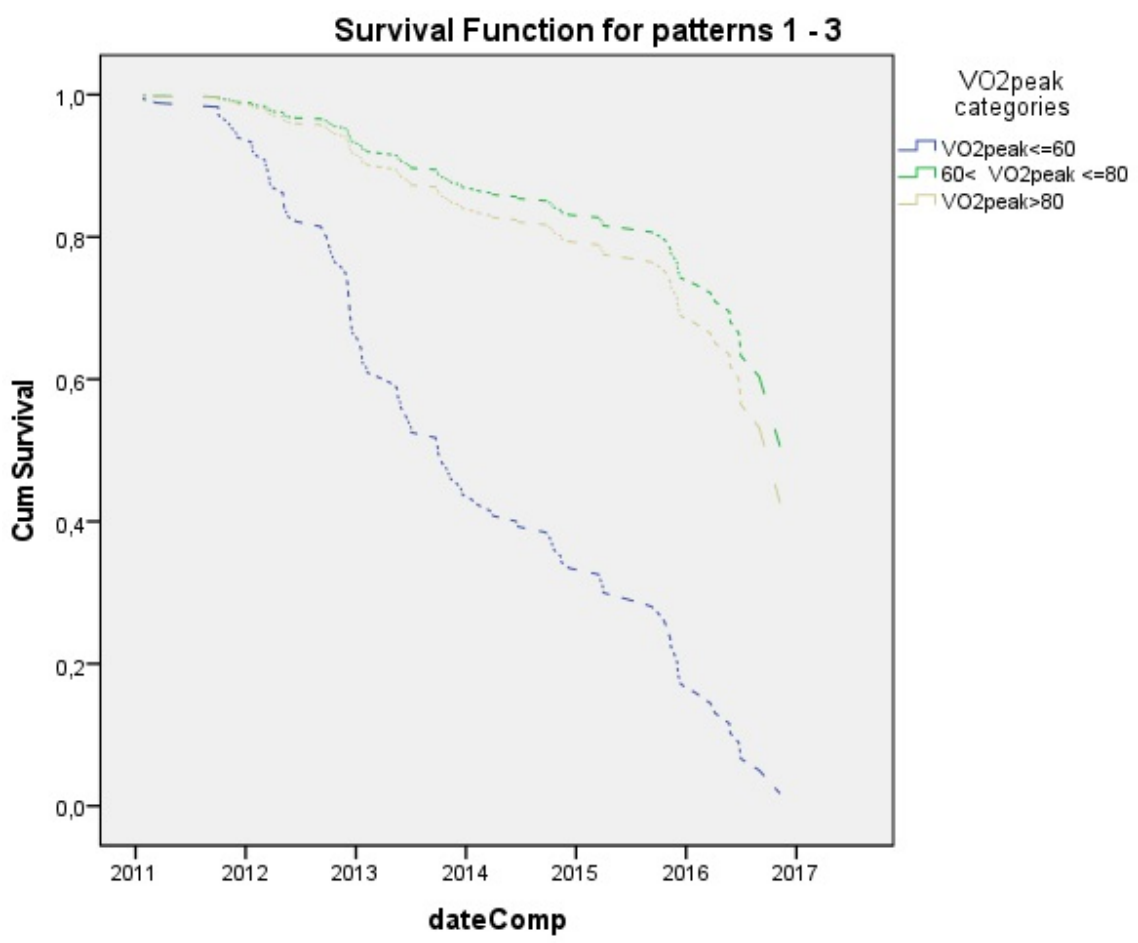

Survival Function for patterns 1 - 3

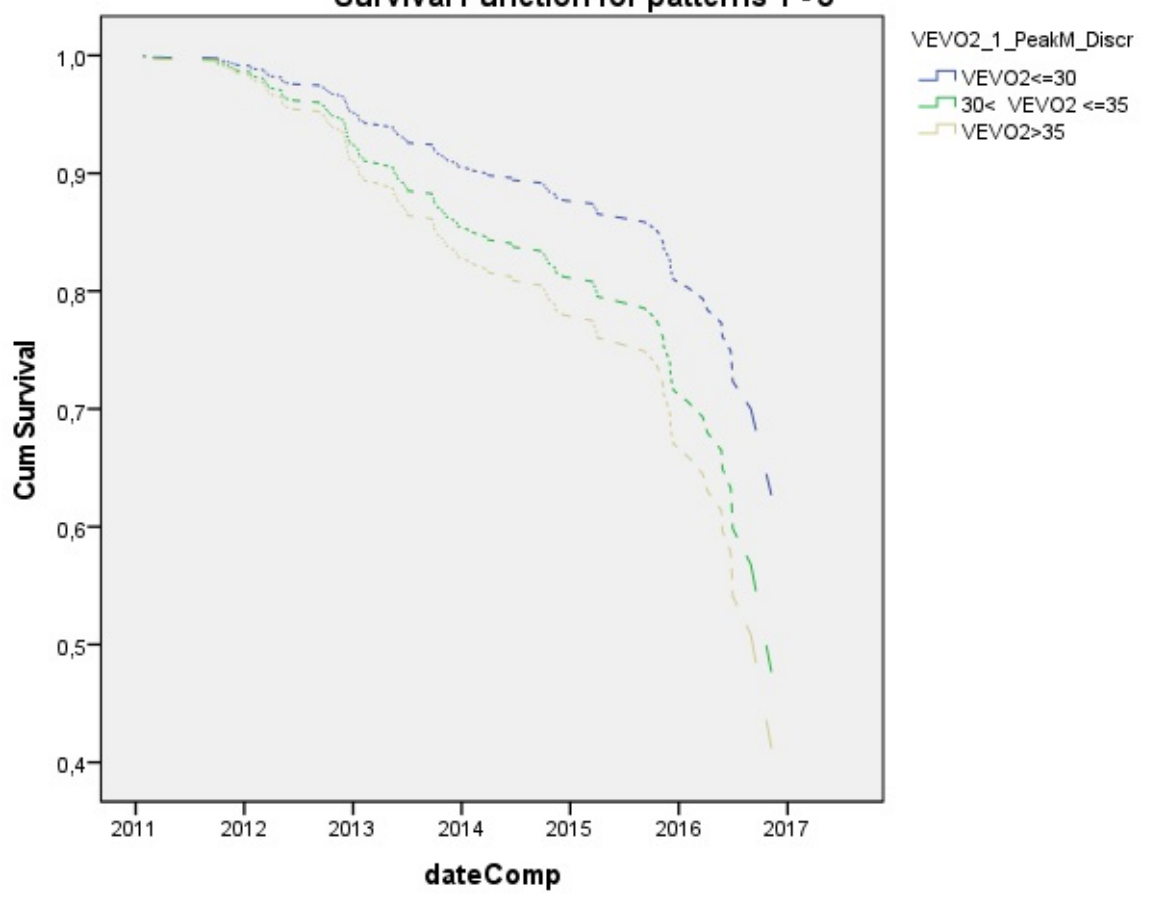




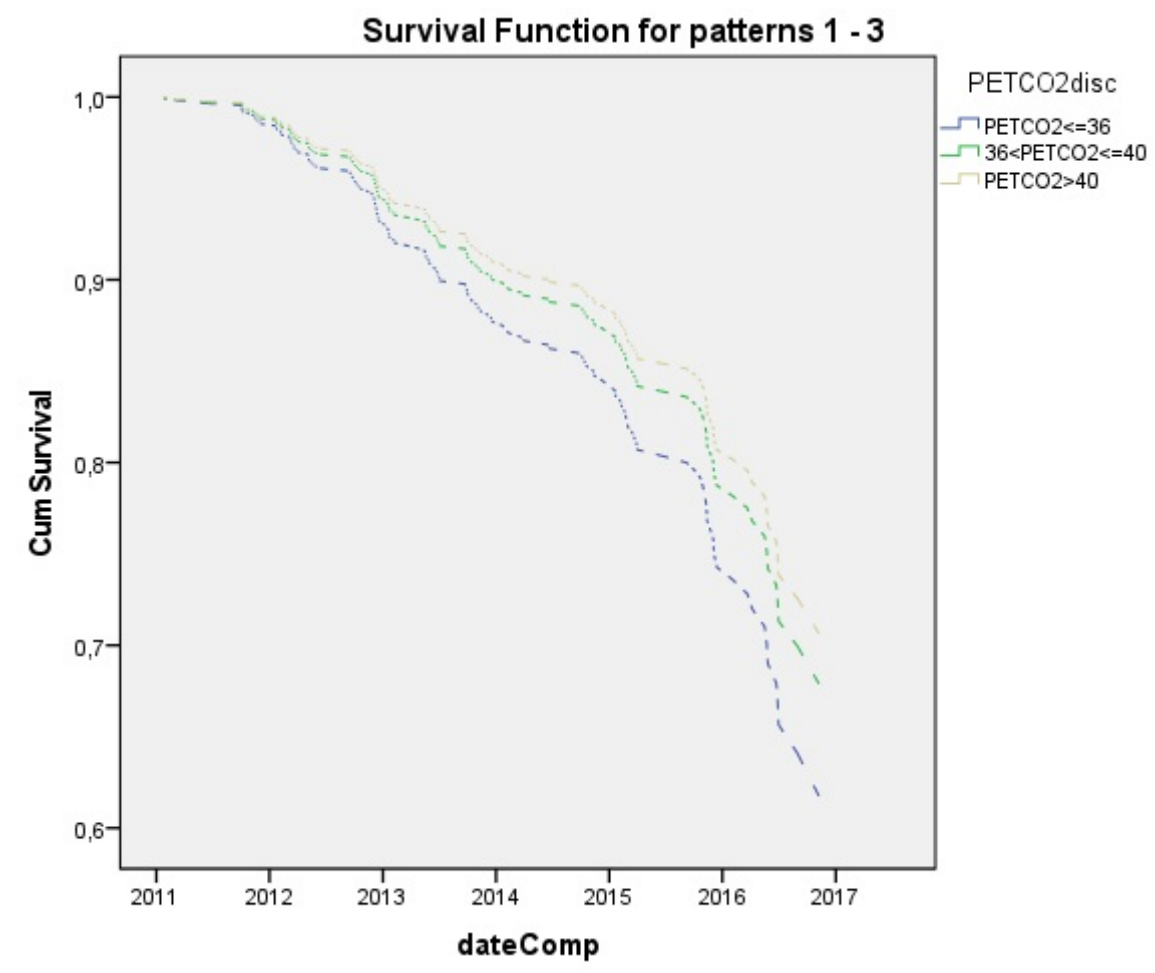

\section{Hosted file}

Table 1.pdf available at https://authorea.com/users/368753/articles/487796-the-fitter-thebetter-cardiopulmonary-exercise-testing-can-predict-pulmonary-exacerbations-in-cysticfibrosis

\section{Hosted file}

Table 2.pdf available at https://authorea.com/users/368753/articles/487796-the-fitter-thebetter-cardiopulmonary-exercise-testing-can-predict-pulmonary-exacerbations-in-cysticfibrosis 